\title{
New Lymphology Combined with Lymphatic Physiology, Innate Immunology, and Oncology
}

\author{
Ohhashi $\mathrm{T}^{1^{\star}}$ and Kawai $\mathrm{Y}^{2}$ \\ ${ }^{1}$ Department of Innovation of Medicine and Health Sciences Research, Shinshu University School of Medicine, Japan \\ ${ }^{2}$ Department of Physiology, Shinshu University School of Medicine, Japan
}

"Corresponding author: Toshio Ohhashi, Department of Innovation of Medicine and Health Sciences Research, University School of Medicine, Japan, Fax: +81263365149; Tel: +81263313071; Email: ohhashi@shinshu-u.ac.jp

Rec date: July 23, 2014, Acc date: August 20, 2014, Pub date: 22 August, 2014

Copyright: (c) 2014 Toshio Ohhashi et al. This is an open-access article distributed under the terms of the Creative Commons Attribution License, which permits unrestricted use, distribution, and reproduction in any medium, provided the original author and source are credited.

Keywords: New lymphology; Lymphatic physiology; Innate immunology; Oncology; Lymphatic networks

\section{New Lymphology Combined with Lymphatic Physiology, Innate Immunology and Oncology}

In more than 85 year-old history of the Gordon Research Conference, a conference on the scheme of lymphatic system was held for the first time on March 2004, in Ventura, California, USA. Such trends of lymphatic Gordon conference are considered to reflect rapid progress of lymphology inspired by the discovery of new markers of lymphatic endothelial cells such as LYVE-1, podoplanin, and Prox1, and then the development of the VEGF C/D and VEGF R3-mediated lymphangiogenesis and lymphatic metastasis of carcinoma cells.

As one of lymphatic functions, it is well known that transport and drainage of the substances through the lymph systems play pivotal roles in keeping to homeostasis of internal environment between the cells in tissues in collaboration with the exchange of substances through blood capillaries and venules, A large number of achievements of the classical lymphology have been mainly accumulated in anatomy [1]. While the functions of lymphatic system have been studied by the many investigators of microcirculation, i.e., Courtice FC et al. [2], Földie M et al. [3], Casley-Smith JR and Foldie M [4], Roddie IC [5], Schmid-Schoebein GW [6], Ohhashi T et al. [7].

On the other hand, it is well known that initial signs of main diseases such as inflammation, tumor, and circulatory disorders, appear as functional abnormalities of the internal environment in tissues. When the internal environment is evaluated from the biological defense points of view, these pathophysiological functions are worked by immunological elements with transport and drainage actions of lymphatic system. When the classical lymphology is viewed again from the physiological and pathophysiological significance, the new lymphology may be the royal road of its final goal by the combination with new lymphatic physiology, innate immunology, and oncology. Thus the new obtained physiological findings are summarized as follows:

(i) There are marked heterogeneity in lymph dynamic functions, which may be related to gravity and lymph flow volume [7], i.e. heartlike spontaneous contractions are observed physiologically in mesenteric and lower extremities' collecting lymph vessels, but not in facial and neck ones with lymphatic valves [8] (ii) The lymph vessels work physiologically under the circumstance of lower oxygen tension such as $30-35 \mathrm{mmHg}[7,9]$. Thus oxygen radicals such as nitric oxide (NO) and prostaglandins play as main regulators for controlling the functions of lymphatic endothelial cells and smooth muscles $[7,10]$ (iii)
The lymph flow (shear stress stimulation) also play important roles in the production and release of NO $[7,11]$ or the gene expression of endothelial constitutive NO synthase [12] (iv) The concentration of albumin in lymph is condensed through the walls of small lymph vessels [13] and lymph nodes [14,15], which may regulate nonselctively the excretion of lymphocytes including natural killer cells from the regional lymph nodes [16] (v) The lymph vessels and lymph nodes are surrounded with adipose tissues [1].

Taking into consideration of the new findings, we have suggested the concept of new lymphology that new more interesting findings and concept will be easily produced in the field of immunology and oncology in connection with lymphatic physiology if the abovementioned findings were combined with the research of innate immunology and sentinel lymph node concept or lymphatic metastasis concept of carcinoma cells in oncology.

In this special commentary, concerning the possibility of establishing new lymphology as a key scientific discipline integrating lymphatic physiology, innate immunology, and oncology, we would like to demonstrate comprehensively our latest studies related with suggesting new lymphology, hoping for the readers to evaluate this possibility.

In the field of lymphatic physiology, in lower animals, particularly amphibia, the flow of lymph is maintained by rhythmically beating lymph hearts. Although there is no lymph heart in the mammal, all lymphatics other than initial lymphatic networks contain smooth muscles in their walls [2]. It has been reported that mammalian mesenteric lymphatics and the collecting lymph vessels of the lower extremities, at least in certain species included human beings, show spontaneous active contractions which are capable of promoting lymph propulsion [17]. We also succeeded in recording action potentials from bovine mesenteric lymphatics simultaneously with phasic contraction waves which had one-to-one correspondence to the action potentials [18]. Calcium current played a major role in producing spike discharge in lymphatic smooth muscles [19]. The activity of spontaneous contractions is also supported with the histological evidence that there exists the presence of vasa vasorum within the media of the well-developed smooth muscle layers of bovine mesenteric lymphatics [20]. The adipose tissue around lymph vessels and lymph nodes may play a pivotal role in keeping blood circulation in the adventitial which distributes enough blood flow through the vasa vasorum within the media. For examples for suggesting the possibility of new lymphology, surgical dissection of the adipose tissue around lymph vessels may prevent the blood circulations through the vasa vasorum, resulting in disappearance of active spontaneous contractions of lymphatic smooth muscles and then disturbance of 
active lymph transport. The presence of vasa vasorum within the media may also reflect the relatively high oxygen requirements of the lymphatic smooth muscles and relatively low oxygen supply from the lymph flow through the lymph vessels. In compatible with this expectation, we have confirmed that the oxygen pressure of lymph in canine thoracic duct is around $35 \mathrm{mmHg}$ [7]. In the case of tumor tissues, the oxygen pressure of the interstitial fluid or lymph in the small lymph vessels is known to be less than $10 \mathrm{mmHg}$ [21].

Taken into consideration of the above mentioned backgrounds of hypoxic physiological condition at the lymph through collecting lymph vessels and lymph nodes, it may be reasonable to consider that oxygen radicals, such as nitric oxide (NO) and reactive oxygen spieces (ROS) may play important roles in the regulation of the spontaneous contractions-mediated active lymph transport in physiological or pathophysiological conditions. Thus, lymphatic endothelial cells, as well as arterial and venous endothelial cells, have the potential generation and release of endogenous NO [22]. The endogenous NO is confirmed to release from lymphatic endothelial cells by the stimulation of not only acetylcholine but also lymph flow (shear stress on the endothelial cells) [23-25]. In fact, the dense immune reactivity of endothelial constitutive (ec) NO synthase (ecNOS) and inducible NOS (iNOS) are confirmed in the cultured canine lymphatic endothelial cells [26]. Recently, we have demonstrated that human lymphatic endothelial cells (LEC) release NO in response to shear stress and genetically regulate the shear stress-mediated control of ecNOS content of the cells. Thus, shear stress-mediated ecNOS expression may play important roles in modulating the sensitivity of human LEC to changes in the lymph flow rate [12].

In addition, in the field of oncology, it is well known that carcinoma, which is an epithelial-origined tumor, is likely to metastasize lymphogenously and that sarcoma, a non-epithelial-origined tumor, and is likely to metastasize hematogenously. However, there has been little systemic research to clarify the mechanisms of lymphatic metastasis of carcinoma cells focusing on the functional properties of tissue spaces (internal environment), relationship between the expression of molecular markers on the carcinoma cells and lymphatic metastasis of the cells, lymph flow-mediated interactions between lymphatic endothelial cells and carcinoma cells, or lymph dynamic analysis of carcinoma cells through the lymph vessels and lymph nodes. However, the clinical impact of the sentinel lymph node (SLN) concept has become one of the most important topics in surgical oncology in patients with breast cancer and melanoma [27]. Recently, gastric cancer has also been identified as a target for SN navigation surgery (SNNS). We attempted to evaluate the usefulness of the contrast-enhanced ultrasound (CEUS)-guided method with Sonazoid for imaging of the lymphatic channels and the SLN of stomach in a porcine model. Contrast imaging using the intragastric or transcutaneous CEUS-guided method with Sonazoid enabled us to produce clear images of the afferent lymph vessel and SLN of the stomach until $2 \mathrm{hr}$ after the injection of Sonazoid. Intranodal flow of the microbubble agent could be also clearly identified using tissue linear harmonic images of the SLN [28].

However, it remains unclear which factors play pivotal roles in deciding which lymph node becomes the SLN. We have considered the SLN to be constantly subjected to a high lymph flow rate. We reached the conclusion in the above-mentioned ultrasound study [28] in which a high lymph flow rate was detected within the SLN of the stomach using the CEUS-guided method with Sonazoid. Therefore, we investigated the effects of shear stress stimulation on the expression of adhesion molecules on human LEC isolated from the afferent lymphatics nearest the SLN of breast cancers, and on the release of shear stress-mediated ATP from human LEC. Thus, the shear stress stimulation produced a significant increase of ICAM-1 expression at proteins and mRNA levels in human LEC. The shear stress-mediated increase of ICAM-1 expression accelerated the attachment of carcinoma cells to human LEC. In conclusion, we have confirmed that shear stress stimulation induces ICAM-1 expression on human LEC by activating cell surface F1/FO ATP synthase, which might contribute the development of a pre metastatic environment within the SLN [29].

Finally, in the field of innate immunology, the lymphatic system has been known to cooperate with lymph nodes and support the biological defense mechanisms, but it has not fully emerged to the forefront of immunology or inflammatology. This is partly related to the evidence that molecular biology and molecular genetics primarily dealing with lymphocytes, cytokines, and immunoglobulins have been regarded as the mainstay of immunological research, but the dynamics of the movement of lymphocytes and lymph through the lymph vessels and lymph nodes have been overlooked in immunology. In addition, the technical difficulty of relative evaluation of the dynamic movement of lymphocytes, the lymph flow rate and the concentration of protein in lymph has contributed to the lack of attention for the interaction with innate immunology and lymph flow dynamics.

However, from the 1950's time the lymphatic system is known to be vital for fluid homeostasis, recirculation of plasma protein, and immune surveillance under condition of health [30]. Thus, most of plasma protein, especially albumin, is known to be filtered through venules into interstitial spaces, which is returned through the lymphatic system to the blood circulation [31]. In addition, it is well established that lymph nodes have a condensing effect of the protein in lymph. Recently, we have also demonstrated that small, water-soluble substances less than 4,400 at molecular weight, but not albumin, were able to pass through the small-sized lymph vessel walls, resulting in condensing effect of albumin in lymph [13]. One of physiological and pathophysiological meanings of the recirculation of plasma protein through the lymphatic system and the condensing effect of albumin in lymph through the lymphatic vessel walls and lymph nodes may be, in part, related to the regulation of innate immunity, which may be compatible with the findings that the non-selective secretion of lymphocytes from lymph nodes displayed a positive correlation with the albumin concentration in lymph with its afferent lymph vessels [16].

However, the precise mechanisms of the condensing effect of lymph vessel walls and the modulating effects of inflammatory cytokines on the condensing effects through the lymph vessel walls remain unknown. Recently, we have demonstrated that the human LEC layer plays key roles in the transport of hydrophilic substances through collecting lymph vessel walls, and that TNF- $\alpha$ or IL- $1 \beta$ significantly increases the permeability of the layers to 4 and $12 \mathrm{kDa}$ dextrans via Rho kinase activation and the ERK $1 / 2$ phosphorylation-mediated organization of F-actin in the LEC [32].

Taken into consideration of the above-mentioned research, we have been proposing the new lymphology combined with cardiovascular physiology, innate immunology, and oncology from the lymphdynamics points of view. We have believed that these lymphatic research subjects combined with functional properties of lymph circulation are considered to include of lymphatic research themes that will contribute to the establishment of new lymphology. 


\section{References}

1. Kotani M (2012) Seven mysteries of lymphatics (in Japanese) Rinpagaku. The official journal of Japanese Society of Lymphology 35: 66-80.

2. Yoffey, Mendel J, Courtice FC (1970) Lymphatics, lymph and the lymph myeloid complex. Academic Press, London.

3. Ruszunyak I, Földi M, Szabo G (1967) Lymphatics and lymph circulation. Pergamen Press, Oxford.

4. Földi M, Casley-Smith JR (1983) Lymphangiology. Schattauer Verkig, Stuttgart.

5. Roddie IC (1990) Lymph transport mechanisms in peripheral lymphatics. News Physiol Sci 5: 85-89

6. Schmid-Schoebein GW (1990) Microlymphatics and lymph flow. Physiol Rev 70: 987-1028.

7. Ohhashi T, Mizuno R, Ikomi F, Kawai Y (2005) Current topics of physiology and pharmacology. Pharma Ther 105: 165-188

8. Hargens AR, Zweifach BM (1977) Contractile stimuli in collecting lymph vessels. Am J Physiol 233: H57-H65.

9. Tsai AG, Johnston PC, Intaglietta M (2003) Oxygen gradients in the microcirculation. Physiol Rev 83: 933-963.

10. Johnston MG, Gordon JL (1981) Regulation of lymphatic contractility by arachidonate metabolites. Nature 293: 294-297.

11. Gashev AA, Davis MJ, Zawieja DC (2002) Inhibition of active lymph pump by flow in rat mesenteric lymphatics and thoracic duct. J Physiol (Lond) 540: 1023-1037.

12. Kawai Y, Yokoyama Y, Kaidoh M, Ohhashi T (2010) Shear stress-induced ATP-mediated endothelial constitutive nitric oxide synthase expression in human lymphatic endothelial cells. Am J Physiol 298: C647-C655.

13. Ono N, Mizuno R, Ohhashi T (2005) Effective permeability of hydrophilic substances through walls of lymph vessels: Roles of endothelial barrier. Am J Physiol Heart and Circ 289: H1676-H1682.

14. Adair TH, Moffatt DS, Paulsen A (1982) Quantitation of changes in lymph protein concentration during lymph node transit. Am J Physiol 243: H351H359.

15. Quin JW, Shannon AD (1977) The influence of the lymph node on protein concentration of efferent lymph leaving the node. J Physiol (Lond) 264: 307-321.

16. Knox P, Pflug JJ (1983) The effect of the canine popliteal node on the composition of lymph. J Physiol (Lond) 345: 1-14.

17. Ohhashi Y, Azuma T, Sakaguchi M (1980) Active and passive mechanical characteristics of bovine mesenteric lymphatics. Am J Physiol 239: H88H95.
18. Ohhashi T, Azuma T, Sakaguchi M (1978) Transmembrane potentials in bovine lymphatic smooth muscle. Proc Soc Exp Biol Med 159: 350-352.

19. Azuma T, Ohhashi T, Sakaguchi M (1977) Electrical activity of lymphatic smooth muscles. Proc Soc Exp Biol Med 155: 270-273.

20. Ohhashi T, Fukushima S, Azuma T (1977) Vasa vasorum within the media of bovine mesenteric lymphatics. Proc Soc Exp Biol Med 154: 582-586.

21. Jain RK (1997) Delivery of molecular and cellular medicine to solid tumors. Microcirculation 4: 1-23.

22. Ohhashi T, Takahashi N (1991) Acetylcholine-induced release of endothelium-derived relaxing factor from lymphatic endothelial cells. Am J Physiol 260: H1172-H1178.

23. Yokoyama S, Ohhashi T (1993) Effects of acetylcholine on spontaneous contractions in isolated bovine mesenteric lymphatics. Am J Physiol Heart Circ Physiol 264: H1460-H1464.

24. Shirasawa Y, Ikomi F, Ohhashi T (2000) Physiological roles of endogenous nitric oxide in lymphatic pump activity of rat mesentery in vivo. Am J Physiol Gastrointest Liver Physiol 278: G551-G556.

25. Tsunemoto H, Ikomi F, Ohhashi T (2003) Flow-mediated release of nitric oxide from lymphatic endothelial cells of pressurized canine thoracic duct. Jpn J Physiol 53: 157-163.

26. Nojiri H, Ohhashi T (1999) Immunolocalization of nitric oxide synthase and VEGF receptors in cultured lymphatic endothelial cells. Microcirculation 6: 75-78.

27. Morton DL, Wen DR, Wong JH, Economou JS, Cagle LA et al. (1992) Technical details of intraoperative lymphatic mapping for early stage melanoma. Arch Surg 127: 392-399.

28. Kawai Y, Ajima K, Nagai T, Kaidoh M, Ohhashi T (2011) Real-time imaging of the lymphatic channels and sentinel lymph nodes of the stomach using contrast-enhanced ultrasonography with Sonazoid in a porcine model. Cancer Sci 102: 2073-2081.

29. Kawai Y, Kaidoh M, Yokoyama Y, Ohhashi T (2012) Pivotal roles of shear stress in the microenvironmental changes that occur within sentinel lymph nodes. Cancer Sci 103: 1245-1252.

30. Wasserman K, Mayerson HS (1951) Exchange of albumin between plasma and lymph. Am J Physiol 165: 15-26.

31. Mehta D, Malik AB (2006) Signaling mechanisms regulating endothelial permeability. Physiol Rev 86: 279-367.

32. Kawai Y, Kaidoh M, Yokoyama Y, Ohhashi T (2014) Pivotal roles of lymphatic endothelial cell layers in the permeability to hydrophilic substances through collecting lymph vessel walls: Effects of inflammatory cytokines. Lymphat Re 